\title{
Guest Editor's Foreword
}

This special issue contains a selection of papers from the 19th ACM Symposium on Computational Geometry, which was held on June 8-10, 2003 in San Diego (USA). The ACM Symposium is the main conference in the area of computational geometry, featuring high-quality papers on topics ranging from combinatorial geometry and computational topology to geometric data structures and applications. For this special issue, seven papers were selected from the more theoretical contributions, presenting results on a variety of topics.

Balogh et al. consider the following problem: what is the maximum number of turns on a monotone path in an arrangement of $n$ lines in the plane? Trivially, the total number of vertices of the arrangement, which is $O\left(n^{2}\right)$, is an upper bound on this number. So far, the best known lower bound was significantly smaller, namely $\Omega\left(n^{7 / 4}\right)$. Balogh et al. show an $\Omega\left(n^{2-(d / \sqrt{\log n})}\right)$ bound, where $d>0$ is a constant, thereby almost settling this long-standing problem.

Chan considers the problem of bounding the worst-case ratio of the weight of a Euclidean minimum spanning tree on a set of points in the plane to the weight of a minimum spanning tree on those points with maximum degree 3 or 4 . By a clever argument he shows that these ratios are bounded by 1.402 and 1.143, respectively, which is the first progress on this problem since 1994. He also obtains improved results in higher dimensions.

Another discrete-geometry problem is considered by Matoušek and Wagner, who study weak $\varepsilon$-nets. $\varepsilon$-Nets and weak $\varepsilon$-nets form an important concept in discrete geometry, with applications in both discrete and computational geometry. Matoušek and Wagner give a beautiful alternative proof of the fact that any finite point set in $\mathbb{R}^{d}$ admits a weak $\varepsilon$-net of size $O\left((1 / \varepsilon)^{d}\right.$ polylog $\left.(1 / \varepsilon)\right)$. They use the new proof to obtain improved bounds on the size of weak $\varepsilon$-nets in various special cases, and to give an efficient algorithm to construct weak $\varepsilon$-nets.

Czumaj and Zhao study fault-tolerant spanners for Euclidean graphs. They prove that any set of $n$ points in $d$-dimensional space admits a $k$-fault-tolerant $t$-spanner of maximum degree $O(k)$ and maximum cost $O\left(k^{2} \cdot W_{\mathrm{MST}}\right)$, where $W_{\mathrm{MST}}$ is the cost of the minimum spanning tree. This result is optimal, and improves both the best known bound on the maximum degree and the best known bound on the maximum cost of fault- 
tolerant spanners. They also present an efficient algorithm for constructing low-degree and low-cost fault-tolerant spanners.

Cole-McLaughlin et al. study a problem from computational topology concerning Reeb graphs of 2-manifolds. Reeb graphs express the connectivity of level sets, and are used in various applications. They obtain several fundamental results in this area: they give tight upper and lower bounds on the number of loops in Reeb graphs both for orientable and non-orientable 2-manifolds, and they give an $O(n \log n)$ algorithm to construct the Reeb graph of a given 2-manifold.

Giesen and Wagner deal with another computational-topology problem, namely manifold learning: construct a model of an unknown $k$-dimensional manifold in $\mathbb{R}^{d}$, given a set of $n$ sample points on the manifold. This is a generalization of the surface-reconstruction problem, where one want to construct a two-dimensional manifold from a set of sample points in $\mathbb{R}^{3}$. Most surface-reconstruction algorithms use the Delaunay triangulation of the sample points. This is not suitable for manifold learning in high dimensions, since the complexity of the Delaunay triangulation is exponential in the dimension. Giesen and Wagner therefore introduce the so-called adaptive neighborhood graph, which has complexity $O\left(n^{2}\right)$ in any dimension, and they prove that, under certain conditions, the adaptive neighborhood graph is guaranteed to be similar to the underlying manifold.

Finally, Har-Peled and Varadarajan study the following shape-fitting problem: how close is a given set $P$ of $n$ points in some high-dimensional space to being collinear? In other words, one wants to compute the minimum-radius cylinder containing all the points. They consider the case where $d$ is not a constant, and can be as large as $n$. The exact version of this problem is NP-hard. Har-Peled and Varadarajan present an $\varepsilon$-approximation algorithm, which runs in time linear in $n$ and $d$. They extend the result to the case where one wants to decide how close the point set is to lying on a $k$-flat.

I wish to close by thanking the authors for submitting their papers to the special issue, the referees for their timely and careful reviews, and Ricky Pollack for giving me the opportunity to edit this special issue. I hope that the readers will find the papers in it valuable and, most of all, that they will enjoy reading them.

Mark de Berg

TU Eindhoven The Netherlands 